PROCEEDINGS OF THE

AMERICAN MATHEMATICAL SOCIETY

Volume 136, Number 6, June 2008, Pages 2019-2029

S 0002-9939(08)09104-1

Article electronically published on February 14, 2008

\title{
SPECTRAL DOMINANCE AND COMMUTING CHAINS
}

\author{
BICH T. HOAI, CHARLES R. JOHNSON, AND ILYA M. SPITKOVSKY \\ (Communicated by Joseph A. Ball)
}

\begin{abstract}
A positive semidefinite (PSD) operator $A$ "spectrally dominates" a PSD operator $B$ if $A^{t}-B^{t}$ is PSD for all $t>0$. We (i) give a new characterization of spectral dominance in finite dimensions in terms of a monotonic chain of intermediate, pairwise commuting operators and (ii) determine for which pairs $A, B$ spectral dominance persists under the taking of arbitrary compressions. Earlier results about spectral dominance are proven (in finite dimensions) in new ways, and several corollary observations are made.
\end{abstract}

\section{INTRODUCTION}

Let $\mathfrak{H}$ be a Hilbert space, and let $A, B$ be selfadjoint bounded operators acting on $\mathfrak{H}$. We say $A \succcurlyeq B$ if $A-B$ is positive semidefinite (PSD for short), the standard Löwner partial order on the real space of selfadjoint operators. In case $\operatorname{dim} \mathfrak{H}=$ $n<\infty$ we will without saying identify $\mathfrak{H}$ with $\mathbb{C}^{n}$, and represent $A, B$ as $n$-by- $n$ matrices: $A, B \in \mathbb{C}^{n \times n}$.

Recall that if $f$ is a monotonic function on $\mathbb{R}$ and $A \succcurlyeq B$, then

$$
f(A) \succcurlyeq f(B)
$$

may not hold. The functions $f$ defined on $[0, \infty)$ and such that (1) holds for any PSD operators (respectively, $n$-by- $n$ matrices) $A, B$ satisfying $A \succcurlyeq B$ are called monotone operator functions (monotone matrix functions of order $n$ ); see [3], [10, Chapter 6], or [1, Chapter 5]. More recent results and references on the subject can be found in 12 .

It is known that $f(x)=x^{t}$ with $0 \leq t \leq 1$ is a monotone operator function (giving the so-called Löwner-Heinz inequality from (1)), while for $t>1$ it is not a monotone matrix function of any order $n \geq 2$.

A dual approach is to consider conditions to be imposed on the PSD pair $A, B$ so that (1) holds for $f(x)=x^{t}$ for any $t>0$, or more generally, for any $f$ nondecreasing on $[0, \infty)$. As it happens, these requirements are equivalent. More specifically, the following result can be extracted from [11; see also [4. Notation $P_{x}$ in its statement means the value of the spectral function on $[x, \infty)$.

Received by the editors November 30, 2006, and, in revised form, January 3, 2007.

2000 Mathematics Subject Classification. Primary 47A63, 15A57, 15A27.

Key words and phrases. Semidefinite operators/matrices, spectral order, power dominance.

The work on this paper in the summer of 2006 was supported in part by the National Science Foundation Grant No. DMS-0353510.

The third author (IMS) is also partially supported by the National Science Foundation Grant No. DMS-0456625. 
Theorem 1. The following statements about PSD operators $A, B$ are equivalent:

(i) $P_{x}(A) \succcurlyeq P_{x}(B)$ for all $x \in \mathbb{R}$,

(ii) $A^{t_{k}} \succcurlyeq B^{t_{k}}$ for some unbounded sequence $t_{k}(>0)$,

(iii) $f(A) \succcurlyeq f(B)$ for any function $f$ non-decreasing on $\mathbb{R}_{+}$.

If condition (i) of Theorem 1 holds for a pair of selfadjoint operators $A, B$, we will write $A \succcurlyeq B$ and say that $A$ spectral dominates $B$. Due to Theorem 1, in case of PSD $A, B$ we will alternatively use the term power dominance. It is clear that $\succcurlyeq^{S}$ is a partial order, stronger than $\succcurlyeq$.

We are interested in two new aspects of spectral dominance. In Section 2, we give an alternative description of the property $A \succcurlyeq B S$ applicable in the finitedimensional setting. Section 4 pursues the conditions under which spectral dominance is inherited under taking compressions. The intermediate Section 3 contains some additional observations and comments.

\section{Spectral order and commuting Chains}

Throughout this section, we are dealing with the finite-dimensional setting only. Observe that condition (i) of Theorem 1 then simply means that an eigenvector $x$ of $A$ corresponding to its eigenvalue $\lambda$ is orthogonal to an eigenvector $y$ of $B$ corresponding to its eigenvalue $\mu$ whenever $\lambda<\mu$. For convenience of reference we will say that the ordered pair of $n$-by- $n$ PSD matrices $A, B$ satisfying this condition is upward orthogonal.

In what follows, we write $X \smile Y$ if the matrices $X$ and $Y$ commute, and we say that $X_{0}, \ldots, X_{m}$ is a commuting chain (of matrices) if $X_{0} \smile X_{1}, X_{1} \smile$ $X_{2}, \ldots, X_{m-1} \smile X_{m}$.

Theorem 2. Let $A$ and $B$ be $n$-by-n PSD matrices. Denote by $\lambda_{1} \geq \cdots \geq \lambda_{n}$ all the eigenvalues of $A$, and by $x_{1}, \ldots, x_{n}$ an orthonormal set of corresponding eigenvectors. Similarly, let $\mu_{1} \geq \cdots \geq \mu_{n}$ denote the eigenvalues of $B$, with $y_{1}, \ldots, y_{n}$ being an orthonormal set of corresponding eigenvectors. Then, the following are equivalent:

(i) $A^{t_{k}} \succcurlyeq B^{t_{k}}$ for some unbounded sequence $t_{k}(>0)$;

(ii) $x_{j}^{*} A^{t} x_{j} \geq x_{j}^{*} B^{t} x_{j}$ for all $t \geq 0$ and $j=1, \ldots, n$;

(iii) $y_{j}^{*} A^{t} y_{j} \geq y_{j}^{*} B^{t} y_{j}$ for all $t \geq 0$ and $j=1, \ldots, n$;

(iv) the pair $A, B$ is upward orthogonal;

(v) there exist PSD matrices $C_{1}, \ldots, C_{k}$ such that $A \succcurlyeq C_{1} \succcurlyeq C_{2} \succcurlyeq \ldots \succcurlyeq C_{k} \succcurlyeq$ $B$ is a commuting chain; and

(vi) for every non-decreasing function $f$ defined on $\mathbb{R}_{+}$, we have $f(A) \succcurlyeq f(B)$.

Of course, the equivalence of statements (i), (iv) and (vi) in Theorem 2 follows directly from Theorem 1 We nevertheless give here an independent proof of all the equivalences in Theorem 2 because it is simpler and allows us to incorporate the additional statements (ii), (iii), (v) smoothly in the flow of the proof.

The subtle part of the proof is the implication (iv) $\longrightarrow(\mathrm{v})$. It uses the following auxiliary result.

Lemma 3. Let the pair $A, B$ be upward orthogonal. Then:

(i) $\lambda_{i} \geq \mu_{i}$ for all $i=1, \ldots, n$ and 
(ii) there exists a matrix $C \succcurlyeq B$ and commuting with $B$ such that the pair $A, C$ is upward orthogonal and unitarily reducible.

Proof. (i) Suppose that $\lambda_{i}<\mu_{i}$ for some $i$. Then $\lambda_{j}<\mu_{k}$ for all $j=i, \ldots, n$ and $k=1, \ldots, i$, so that the $i$-dimensional span of $y_{1}, \ldots, y_{i}$ is orthogonal to the $(n-i+1)$-dimensional span of $x_{i}, \ldots, x_{n}$. This is an impossibility.

(ii) Let $k$ be the smallest index such that $\lambda_{n} \geq \mu_{k}$ (such a $k$ exists due to the already-proved part of the lemma). Let $C$ be the Hermitian matrix with the same eigenvector basis as $B$ and the eigenvalues

$$
\nu_{j}= \begin{cases}\mu_{j} & \text { if } j<k \\ \lambda_{n} & \text { otherwise. }\end{cases}
$$

It is clear that $C$ commutes with $B$ and $C \succcurlyeq B$. Since $\nu_{j}>\lambda_{k}$ if and only if $\mu_{j}>\lambda_{k}$, the pair $A, C$ is upward orthogonal as is the pair $A, B$. On the other hand, the "upward orthogonality" of the pair $A, B$ and our choice of $k$ imply that the eigenvector $x_{n}$ of $A$ is orthogonal to the span of $y_{1}, \ldots, y_{k-1}$ and therefore lies in the span of $y_{k}, \ldots, y_{n}$. Since the latter is the eigenspace of $C$ (corresponding to the eigenvalue $\lambda_{n}$ ), the matrices $A$ and $C$ have common eigenvectors and therefore joint non-trivial invariant subspaces.

Proof of Theorem 2. Implications (i) $\longrightarrow$ (ii), (i) $\longrightarrow$ (iii) and (vi) $\longrightarrow$ (i) are obvious. If (v) holds and $f$ is as in (vi), then $f(A) \succcurlyeq f\left(C_{1}\right) \succcurlyeq \ldots \succcurlyeq f\left(C_{k}\right) \succcurlyeq f(B)$, so that (vi) holds. Thus, (v) $\longrightarrow$ (vi).

Expanding $x_{j}$ across the orthonormal basis $\left\{y_{k}\right\}_{k=1}^{n}$, we may rewrite (ii) as

$$
\lambda_{j}^{t} \geq \sum_{k=1}^{n}\left|x_{j}^{*} y_{k}\right|^{2} \mu_{k}^{t}, \quad t \geq 0 .
$$

The latter inequality cannot hold for $t$ sufficiently large, unless $x_{j}^{*} y_{k}=0$ whenever $\mu_{k}>\lambda_{j}$. Thus, (ii) $\longrightarrow$ (iv). The implication (iii) $\longrightarrow$ (iv) can be proved in a similar fashion. It remains to show that (iv) $\longrightarrow$ (v).

Applying Lemma 3, we reduce the general case to the situation in which the pair $A, B$ is unitarily reducible. Since upward orthogonality is preserved under unitary similarity, without loss of generality we may suppose that $A$ and $B$ are block diagonal:

$$
A=\left[\begin{array}{ll}
A_{1} & \\
& A_{2}
\end{array}\right], \quad B=\left[\begin{array}{ll}
B_{1} & \\
& B_{2}
\end{array}\right],
$$

with the square blocks $A_{1}$ and $B_{1}$ (and therefore $A_{2}$ and $B_{2}$ ) of the same size. The pairs $A_{1}, B_{1}$ and $A_{2}, B_{2}$ are upward orthogonal together with the pair (2). A straightforward induction argument (on the size of the matrices under consideration) completes the proof.

\section{FURTHER REMARKS}

3.1. Related inequalities. Among other things, Theorem 1 means that if $A \succcurlyeq^{S} B$, then $A^{p} \succcurlyeq B^{p}$ for all $p \geq 0$. Consequently, $B^{r} A^{p} B^{r} \succcurlyeq B^{p+2 r}$ for all $p, r \geq 0(p \geq 0$, $r \in \mathbb{R}$, if $B$ is invertible), and

$$
\left(B^{r} A^{p} B^{r}\right)^{1 / q} \succcurlyeq B^{(p+2 r) / q}
$$


for $p, r \geq 0, q \geq 1$ ( $p \geq 0, r \in \mathbb{R}, q \geq 1$ if $B$ is invertible) by the Löwner-Heinz inequality. Similarly,

$$
A^{(p+2 r) / q} \succcurlyeq\left(A^{r} B^{p} A^{r}\right)^{1 / q}
$$

for $p, r \geq 0, q \geq 1$ ( $p \geq 0, r \in \mathbb{R}, q \geq 1$ if $A$ is invertible). It is interesting to compare these statements with Furuta's result [5] according to which (3) and (4) hold if $A \succcurlyeq B$ and the parameters satisfy the more restrictive conditions $p, r \geq 0$, $q \geq 1,(1+2 r) q \geq p+2 r$. It is also shown in [5] that the latter condition is essential, even for $2 \times 2$ matrices $A, B$. Naturally, in Furuta's counterexample $A$ does not spectral dominate $B$.

Observe that condition $q \geq 1$ cannot be weakened to $q \geq 0$ even when $A \succcurlyeq^{S} B$. In other words, it is not true that $A \succcurlyeq B$ implies $B^{r} A^{p} B^{r} \succcurlyeq B^{p+2 r}$. The following example illustrates this point for $p=r=1$.

Example. Let

$$
A=\left[\begin{array}{cc}
2+\epsilon & \epsilon \\
\epsilon & 3
\end{array}\right], \quad B=\left[\begin{array}{ll}
1 & 0 \\
0 & 2
\end{array}\right] .
$$

Then for any $\epsilon \in(0,1)$ both eigenvalues of $A$ exceed 2 , so that $A \succcurlyeq B$. On the other hand, for $\epsilon$ small enough the eigenvalues of $B A B$ are close to 2 and 12 while $B^{3}$ has the eigenvalues 1 and 8 . Since $B A B$ and $B^{3}$ do not commute when $\epsilon \neq 0$, there is no spectral dominance relation between them.

See [6] and the references therein for additional classes of operator inequalities equivalent to spectral dominance.

3.2. Explicit construction. The proof of Theorem 2. as given, hides somewhat a nice construction for the inserted, sequentially commuting matrices $C_{i}$, described in (v). For this reason we give an explicit example of how this may be done.

Example. We begin with the interspersal pattern

$$
\lambda_{1}>\lambda_{2}>\lambda_{3}>\mu_{1}=\mu_{2}>\lambda_{4}>\mu_{3}>\lambda_{5}>\mu_{4}>\mu_{5}
$$

for $n$-by- $n$ PSD matrices $A, B$ with eigenvalues and eigenvectors labeled as before. Theorem 2 requires that for $A, B$ to be upward orthogonal, $x_{4}$ must be orthogonal to $y_{1}, y_{2}$ and $x_{5}$ to $y_{1}, y_{2}, y_{3}$. We construct a commuting chain of three matrices between $A$ and $B$. Set

$$
\nu_{j}= \begin{cases}\mu_{j} & \text { if } j<4, \\ \lambda_{5} & \text { otherwise. }\end{cases}
$$

Since $x_{5}$ lies in the span of $y_{4}, y_{5}$ we may set the eigenvector associated with $\nu_{5}=\lambda_{5}$ to be equal to $x_{5}$ and choose some vector $z_{4}$ also contained in the span of $y_{4}, y_{5}$ and orthogonal to $x_{5}$ to be the eigenvector for $\nu_{4}=\lambda_{5}$. Keeping all other associated eigenvectors and eigenvalues in $B$, we have determined $C_{3}$ to be the matrix with eigenvalues and eigenvectors as indicated:

$$
\begin{array}{lllll}
\mu_{1} & \mu_{2} & \mu_{3} & \lambda_{5} & \lambda_{5} \\
y_{1} & y_{2} & z_{3} & z_{4} & x_{5}
\end{array}
$$

$A$ and $C_{3}$ share the eigenvector $x_{5}$ and so are jointly reducible. Thus, in constructing $C_{2}$ we consider only the $n=4$ block matrices determined by the first four eigenvalues and eigenvectors of $A$ and $C_{3}$ after unitary reduction. Since $\mu_{1}, \mu_{2}>\lambda_{3}$, we set $\nu_{3}, \nu_{4}=\lambda_{4}$ and retain all other eigenvalues and eigenvectors. Since the pair 
$A, C_{3}$ is upward orthogonal, $x_{4}$ is orthogonal to $y_{1}, y_{2}$ and to $x_{5}$ by assumption. Then $x_{4}$ lies in the span of $y_{3}, z_{4}$, so we assign $x_{4}$ as the eigenvector associated with $\nu_{4}=\lambda_{4}$ and choose $z_{3}$ orthogonal to $x_{5}$ in the same subspace for eigenvalue $\nu_{3}$. Having determined $C_{2}$, we construct $C_{1}$ in a similar fashion. Noting that $A$ and $C_{2}$ share eigenvectors $x_{4}, x_{5}$ we consider $n=3$. Because $\lambda_{3}>\mu_{1}$, we set $\nu_{1}, \nu_{2}, \nu_{3}=\lambda_{3}$ and keep the other eigenvalues and eigenvectors. The eigenvectors $x_{1}, x_{2}, x_{3}$ lie in the span of $y_{1}, y_{2}, y_{3}$, so we may choose these to be the eigenvectors associated with corresponding $\nu$ 's. The commuting chain of matrices is summarized below.

$\begin{array}{cccccc}B: & \mu_{1} & \mu_{2} & \mu_{3} & \mu_{4} & \mu_{5} \\ & y_{1} & y_{2} & y_{3} & y_{4} & y_{5} \\ & & & & & \\ C_{3}: & \mu_{1} & \mu_{2} & \mu_{3} & \lambda_{5} & \lambda_{5} \\ & y_{1} & y_{2} & z_{3} & z_{4} & x_{5} \\ & & & & & \\ C_{2}: & \mu_{1} & \mu_{2} & \lambda_{4} & \lambda_{4} & \lambda_{5} \\ & y_{1} & y_{2} & z_{3} & x_{4} & x_{5} \\ & & & & & \\ C_{1}: & \lambda_{3} & \lambda_{3} & \lambda_{3} & \lambda_{4} & \lambda_{5} \\ & x_{1} & x_{2} & x_{3} & x_{4} & x_{5} \\ & & & & & \\ A: & \lambda_{1} & \lambda_{2} & \lambda_{3} & \lambda_{4} & \lambda_{5} \\ & x_{1} & x_{2} & x_{3} & x_{4} & x_{5}\end{array}$

By construction, $A \succcurlyeq C_{1} \succcurlyeq C_{2} \succcurlyeq C_{3} \succcurlyeq B$ and $A \smile C_{1}, C_{1} \smile C_{2}, C_{2} \smile C_{3}$, $C_{3} \smile B$. It follows that $A \succcurlyeq B$.

Each application of Lemma 3 decreases the size of the matrices under consideration by (at least) one. Thus, the number $k$ of intermediate matrices $C_{j}$ in condition (v) of Theorem 2 can be chosen not to exceed $n-1$. A better upper bound can be obtained if we first unitarily reduce the pair $A, B$ to the smallest blocks possible. Namely, $k \leq m-1$, where $m$ is the size of the largest block in such a reduction. In particular, for commuting matrices $A$ and $B, m=1$, so that no intermediate matrices are needed at all $(k=0)$. One intermediate matrix suffices if and only if the pair $A, B$ is unitarily similar to the orthogonal sum of pairs $A_{k}, B_{k}$ such that the smallest eigenvalue of $A_{k}$ beats the largest eigenvalue of $B_{k}$. This happens in particular if $\lambda_{n} \geq \mu_{1}$, in which case the intermediate matrix $C$ can be chosen as a scalar multiple of the identity. It also happens if $m=2$, which is guaranteed for example (see [2, 9, or [7]) when $A$ and $B$ belong to a $C^{*}$-algebra generated by two orthogonal projections. On the other hand, even for $n=3$ it is possible that two intermediate matrices are required. This happens when

$$
\lambda_{1}>\lambda_{2}>\mu_{1}>\lambda_{3}>\mu_{2}>\mu_{3},
$$

$x_{3}$ is orthogonal to $y_{1}$ (so that $A \succcurlyeq B$ ) but $A, B$ do not have common eigenvectors and therefore form a unitarily irreducible pair.

Note, further, that from the construction, a number of $C_{i}$ 's equal to $n+1$ less the greatest index $j$ such that $\lambda_{j} \geq \mu_{1}$ is sufficient for $n>1$. In the simultaneously reducible case, an improvement may be achieved. The quantity may be calculated for each pair of corresponding blocks in the finest reduced form, relative to their size, and the largest such quantity is sufficient. The fact that $n=1$ is an exception (no matrices need be inserted) explains that no matrices need be inserted in the 
commuting case. Do these bounds, taken together, give the fewest possible inserted matrices? We know of no counterexample.

3.3. Diagonal entry dominance. Each of parts (ii) and (iii) in the statement of Theorem 2 may be interpreted in terms of a simultaneous unitary similarity on $A$ and $B$ that diagonalizes one of them. For example, (ii) indicates that the diagonal form of $A$ raised to the power $t$ has diagonal entries that beat those of $B^{t}$.

Observe that condition (i) of Theorem 2 in a trivial way implies that

$$
z_{j}^{*} A^{t} z_{j} \geq z_{j}^{*} B^{t} z_{j} \text { for all } t \geq 0 \text { and } j=1, \ldots, n
$$

for any orthonormal basis $z_{j}$ of $\mathbb{C}^{n}$. Implications (i) $\longrightarrow$ (ii) and (i) $\longrightarrow$ (iii) of the theorem are just particular cases of this simple observation when the basis involved consists of the eigenvectors of $A$ or $B$. The next example shows that switching to an arbitrary orthonormal basis in condition (ii) or (iii) does not give an equivalent condition even if, in addition, we suppose that $A \succcurlyeq B$.

Example. Consider the matrices

$$
\begin{aligned}
& A=\left[\begin{array}{ll}
\frac{1}{\sqrt{2}} & \frac{1}{\sqrt{2}} \\
\frac{-1}{\sqrt{2}} & \frac{1}{\sqrt{2}}
\end{array}\right]\left[\begin{array}{ll}
4 & 0 \\
0 & 2
\end{array}\right]\left[\begin{array}{cc}
\frac{1}{\sqrt{2}} & \frac{-1}{\sqrt{2}} \\
\frac{1}{\sqrt{2}} & \frac{1}{\sqrt{2}}
\end{array}\right]=\left[\begin{array}{cc}
3 & -1 \\
-1 & 3
\end{array}\right], \\
& B=\left[\begin{array}{cc}
\frac{3}{5} & \frac{4}{5} \\
\frac{-4}{5} & \frac{3}{5}
\end{array}\right]\left[\begin{array}{ll}
3 & 0 \\
0 & 1
\end{array}\right]\left[\begin{array}{cc}
\frac{3}{5} & \frac{-4}{5} \\
\frac{4}{5} & \frac{3}{5}
\end{array}\right]=\frac{1}{25}\left[\begin{array}{cc}
43 & 3 \\
3 & 57
\end{array}\right] .
\end{aligned}
$$

Direct computations yield that the matrices $A, B$ are PSD, $A \succcurlyeq B$, and

$$
\begin{aligned}
& A^{t}=\frac{1}{2}\left[\begin{array}{cc}
4^{t}+2^{t} & -4^{t}+2^{t} \\
-4^{t}+2^{t} & 4^{t}+2^{t}
\end{array}\right], \\
& B^{t}=\frac{1}{25}\left[\begin{array}{cc}
3^{t+2}+16 & 3^{t+2}-4 \cdot 3^{t+1}+12 \\
3^{t+2}-4 \cdot 3^{t+1}+12 & 16 \cdot 3^{t}+9
\end{array}\right] .
\end{aligned}
$$

Elementary calculus shows that the diagonal entries of $A^{t}-B^{t}$,

$$
\frac{1}{2}\left(4^{t}+2^{t}\right)-\frac{9}{25} 3^{t}-\frac{16}{25} \text { and } \frac{1}{2}\left(4^{t}+2^{t}\right)-\frac{16}{25} 3^{t}-\frac{9}{25}
$$

are monotonically increasing and thus positive functions of $t \geq 0$. However, $A$ does not power dominate $B$. To see this, take for example $t=10$ and obtain (to three decimal places)

$$
A^{10}-B^{10} \approx 10^{5}\left[\begin{array}{cc}
5.035 & -4.954 \\
-4.954 & 4.870
\end{array}\right]
$$

which has eigenvalues, to three decimal places, $9.908 \times 10^{5}$ and -226.924 .

\section{Total SPECTRAL Dominance}

Recall that for any subspace $\mathcal{L}$ of $\mathfrak{H}$, the compression $T_{\mathcal{L}}(A)$ of $A$ onto $\mathcal{L}$ is defined as the linear transformation $P A P$ considered on $\mathcal{L}$, in which $P$ is the orthogonal projection of $\mathfrak{H}$ onto $\mathcal{L}$. If $\mathfrak{H}=\mathbb{C}^{n}$ and $\mathcal{L}$ is the subspace generated by the coordinate vectors $e_{i}$ with $i$ from $\alpha \subset\{1, \ldots, n\}$, then $T_{\mathcal{L}}(A)$ is simply the principal submatrix of $A$ indexed by $\alpha$, the standard notation for which is $A[\alpha]$.

We say that a (partial) order $\geq$ on the set of selfadjoint operators is inherited if $A \geq B$ implies $T_{\mathcal{L}}(A) \geq T_{\mathcal{L}}(B)$ for all subspaces $\mathcal{L}$ of $\mathfrak{H}$. It is well known (and follows directly from the definition) that the order $\succcurlyeq$ is inherited, and it is therefore natural to ask the same question about $\stackrel{S}{\succcurlyeq}$. 
Observe that for one-dimensional $\mathcal{L}$ the compressions $T_{\mathcal{L}}(A)$ and $T_{\mathcal{L}}(B)$ are just numbers. Thus, $T_{\mathcal{L}}(A) \succcurlyeq T_{\mathcal{L}}(B)$ in this case implies $T_{\mathcal{L}}(A) \succcurlyeq T_{\mathcal{L}}(B)$. Consequently, the order $\succcurlyeq^{S}$ is inherited in the case $n=2$.

The following example shows that, starting with $n=3$, inheritance need not hold.

Example. Let $A$ and $B$ be the two PSD matrices below:

$$
\begin{aligned}
& A=\left[\begin{array}{ccc}
\frac{1}{\sqrt{3}} & 0 & \frac{2}{\sqrt{6}} \\
\frac{1}{\sqrt{3}} & \frac{-1}{\sqrt{2}} & \frac{1}{\sqrt{6}} \\
\frac{1}{\sqrt{3}} & \frac{1}{\sqrt{2}} & \frac{1}{\sqrt{6}}
\end{array}\right]\left[\begin{array}{lll}
7 & 0 & 0 \\
0 & 6 & 0 \\
0 & 0 & 3
\end{array}\right]\left[\begin{array}{ccc}
\frac{1}{\sqrt{3}} & \frac{1}{\sqrt{3}} & \frac{1}{\sqrt{3}} \\
0 & \frac{-1}{\sqrt{2}} & \frac{1}{\sqrt{2}} \\
\frac{2}{\sqrt{6}} & \frac{1}{\sqrt{6}} & \frac{1}{\sqrt{6}}
\end{array}\right], \\
& B=\left[\begin{array}{ccc}
\frac{1}{\sqrt{5}} & \frac{2}{\sqrt{30}} & \frac{2}{\sqrt{6}} \\
\frac{2}{\sqrt{5}} & \frac{-1}{\sqrt{30}} & \frac{-1}{\sqrt{6}} \\
0 & \frac{5}{\sqrt{30}} & \frac{-1}{\sqrt{6}}
\end{array}\right]\left[\begin{array}{lll}
5 & 0 & 0 \\
0 & 2 & 0 \\
0 & 0 & 1
\end{array}\right]\left[\begin{array}{ccc}
\frac{1}{\sqrt{5}} & \frac{2}{\sqrt{5}} & 0 \\
\frac{2}{\sqrt{30}} & \frac{-1}{\sqrt{30}} & \frac{5}{\sqrt{30}} \\
\frac{2}{\sqrt{6}} & \frac{-1}{\sqrt{6}} & \frac{-1}{\sqrt{6}}
\end{array}\right] .
\end{aligned}
$$

Keeping our notation from before, we have the interspersal

$$
\lambda_{1}>\lambda_{2}>\mu_{1}>\lambda_{3}>\mu_{2}>\mu_{3}
$$

with the pair $A, B$ upward orthogonal. Then $A \succcurlyeq B$. Consider the submatrices $A[1,2]$ and $B[1,2]$. Direct computations yield

$$
\begin{aligned}
& A[1,2]=\left[\begin{array}{ll}
\frac{13}{3} & \frac{4}{3} \\
\frac{4}{3} & \frac{35}{3}
\end{array}\right], \\
& B[1,2]=\left[\begin{array}{ll}
\frac{29}{15} & \frac{23}{15} \\
\frac{123}{15} & \frac{127}{30}
\end{array}\right] .
\end{aligned}
$$

Because $A \succcurlyeq B$, we know that $A[1,2] \succcurlyeq B[1,2]$. The eigenvalues of $A[1,2]$ are $\widehat{\lambda}_{1} \approx 6.613$ and $\widehat{\lambda}_{2} \approx 3.554$ while those of $B[1,2]$ are $\widehat{\mu}_{1}=5$ and $\widehat{\mu}_{2}=\frac{7}{6}$. This gives the interspersal

$$
\widehat{\lambda}_{1}>\widehat{\mu}_{1}>\widehat{\lambda}_{2}>\widehat{\mu}_{2} \text {. }
$$

As noted previously, in the 2 -by-2 case, for spectral dominance to hold with this pattern of interspersal, $A[1,2]$ must commute with $B[1,2]$. This is not the case.

It therefore makes sense to consider yet another order on the set of selfadjoint operators. Namely, we say that $A$ totally spectral dominates $B$ if for all subspaces $\mathcal{L}$ of $\mathfrak{H}, T_{\mathcal{L}}(A) \succcurlyeq T_{\mathcal{L}}(B)$. Let us also agree to call the selfadjoint operators $A, B$ identity aligned if $A, B, I$ are linearly dependent over $\mathbb{C}$ (equivalently: over $\mathbb{R}$ ).

Theorem 4. A totally spectral dominates $B$ if and only if either (i) the spectrum of $A$ lies to the right of the spectrum of $B$, or (ii) $A \succcurlyeq B$ and $A, B$ are identity aligned.

Of course, condition (ii) implies that the operators $A$ and $B$ commute. In the finite-dimensional setting, Theorem 4 therefore implies the following simpler statement.

Corollary 5. Let the pair of n-by-n matrices $A, B$ be unitarily irreducible. Then $A$ totally spectral dominates $B$ if and only if $\lambda_{n} \geq \mu_{1}$. 
Observe also that for $n=2$ the matrices $A, B$ are identity aligned whenever they commute. Therefore, in this case, Theorem 4 follows directly from the previous discussion of spectral dominance and the fact that for $n=2$ it coincides with total spectral dominance.

The rest of this section is devoted to the proof of Theorem 4 for $n \geq 3$.

Proof of sufficiency. Under condition (i), let us invoke the fact that for any selfadjoint operator $X$ the closure of its numerical range $W(X)$ is the convex hull of its spectrum (see [8, 10 for this and other relevant properties of the numerical range). Consequently, $W(A)$ lies to the right of $W(B)$. This property is inherited by the compressions $T_{\mathcal{L}}(A), T_{\mathcal{L}}(B)$. This in turn implies spectral dominance of $T_{\mathcal{L}}(A)$ over $T_{\mathcal{L}}(B)$.

Under condition (ii), one simply needs to observe that the identity aligned property is inherited by compressions. Thus, for any subspace $\mathcal{L}$, the compressions of $A$ and $B$ onto $\mathcal{L}$ commute. So, $A \succcurlyeq B$ in this setting as well implies not only that $T_{\mathcal{L}}(A) \succcurlyeq T_{\mathcal{L}}(B)$, but in fact $T_{\mathcal{L}}(A) \succcurlyeq T_{\mathcal{L}}(B)$.

The proof of necessity is more involved, and we divide it into several steps. First, we prove it in what seems a very narrow setting.

Lemma 6. Let $A$ and $B$ be commuting 3-by-3 PSD matrices. If $A$ totally power dominates $B$ and $\lambda_{3}<\mu_{1}$, then $A$ and $B$ are identity aligned.

Proof. If one of the matrices $A, B$ is a scalar multiple of the identity, the pair $A, B$ obviously is identity aligned. Therefore, we may suppose that $A, B$ are not scalar multiples of $I$.

Due to the commutativity of $A$ and $B$, we can choose the orthonormal basis consisting of joint eigenvectors of $A$ and $B$. In other words, we may suppose that (in our standard notation of Theorem 2) $y_{1}, y_{2}, y_{3}$ is simply a permutation of $x_{1}, x_{2}, x_{3}$. Since $\lambda_{3}<\mu_{1}, y_{1}$ must be different from $x_{3}$. Relabel the remaining element of the basis $\left\{x_{1}, x_{2}, x_{3}\right\}$ by $z$.

Consider a 2-dimensional subspace $\mathcal{L}$ of $\mathbb{C}^{3}$, denoting by $u$ a unit vector in its orthogonal complement. For $u$ sufficiently close to $z$, the spectra of $T_{\mathcal{L}}(A)$ and $T_{\mathcal{L}}(B)$ will contain points close to $\lambda_{1}$ and $\mu_{3}$, respectively. Consequently, the largest eigenvalue of $T_{\mathcal{L}}(B)$ is greater than the smallest eigenvalue of $T_{\mathcal{L}}(A)$. From here and the total power dominance of $A$ over $B$ it follows that $T_{\mathcal{L}}(A)$ commutes with $T_{\mathcal{L}}(B)$. Since $A$ and $B$ also commute, we conclude that

$$
P A Q B P=P B Q A P,
$$

where $P$ is the orthogonal projection onto $\mathcal{L}$ and $Q=I-P$ is the complementary projection.

Now impose the condition that $u$ is not orthogonal to any of the $x_{j}$, and therefore is not an eigenvector of $A$ or $B$. Then $A \mathcal{L}$ is not a subspace of $\mathcal{L}$, so that the set of $x \in \mathcal{L}$ such that $A x \in \mathcal{L}$ is at most one-dimensional. The same is true for the set of $x \in \mathcal{L}$ such that $B x \in \mathcal{L}$. Then by simple dimension considerations, there is a $v \in \mathcal{L}$ for which $A v, B v \notin \mathcal{L}$, so that $Q A v, Q B v \neq 0$.

Then

$$
Q A v=c_{1} u, \quad Q B v=c_{2} u,
$$

in which $c_{1}, c_{2}$ are non-zero scalars. From here and (9) it follows that

$$
P\left(c_{2} A-c_{1} B\right) u=0 ;
$$


that is, $u$ is an eigenvector of $c_{2} A-c_{1} B$. Due to the choice of $u$, this is possible only when $c_{2} A-c_{1} B$ is a scalar multiple of the identity matrix, that is, when $A$ and $B$ are identity aligned.

Next, an auxiliary statement.

Lemma 7. Let $A$ and $B$ be $n$-by-n matrices such that $A$ totally spectral dominates $B$ while $\lambda_{i}<\mu_{j}$ for some $i, j=1, \ldots, n$. Then $y_{j}$ (an eigenvector of $B$ corresponding to the eigenvalue $\mu_{j}$ ) is also an eigenvector of $A$, and $x_{i}$ (an eigenvector of $A$ corresponding to the eigenvalue $\lambda_{i}$ ) is also an eigenvector of $B$.

Proof. We will prove the statement regarding $y_{j}$; the proof for $x_{i}$ is similar.

Consider a two-dimensional subspace $\mathcal{L}$ spanned by $y_{j}$ and a vector $x$ the choice of which will be discussed later. Then the linear transformation $T_{\mathcal{L}}(B)$ has the diagonal matrix

$$
\left[\begin{array}{cc}
\mu_{j} & 0 \\
0 & \tilde{\mu}
\end{array}\right]
$$

in the orthonormal basis with the first vector $y_{j}$. Choosing $x$ sufficiently close to $x_{i}$ we may guarantee that one of the eigenvalues of $T_{\mathcal{L}}(A)$ is so close to $\lambda_{i}$ that it is smaller than $\mu_{j}$. Since $T_{\mathcal{L}}(A) \succcurlyeq{ }^{S} T_{\mathcal{L}}(B)$, it follows that $\tilde{\mu}<\mu_{j}$ and that $T_{\mathcal{L}}(A) \smile T_{\mathcal{L}}(B)$. Consequently, the vector $A y_{j}$ is orthogonal with $x-y_{j}^{*} x y_{j}$.

Letting $x$ run through a basis of $\mathbb{C}^{n}$ (which can be done while keeping it as close to $x_{i}$ as needed for the reasoning above to be valid), we conclude that $A y_{j}$ is collinear to $y_{j}$.

Note that Corollary 5 is proved at this stage.

Proof of necessity; general finite-dimensional setting. We need only consider pairs $A, B$ satisfying the conditions of Lemma 7 Fix a pair $i, j$ for which $\lambda_{i}<\mu_{j}$, and choose the respective eigenvectors $x_{i}, y_{j}$. The eigenvalue $\tilde{\lambda}$ of $A$ corresponding to the eigenvector $y_{j}$ (we are using Lemma 7 here) is greater than $\mu_{j}$ due to the property $A \succcurlyeq B$, and is therefore different from $\lambda_{i}$. Thus, the vectors $x_{i}, y_{j}$ are orthogonal, and we can introduce a unitary transformation $U$ that maps $e_{1}$ to $x_{i}$, $e_{2}$ to $y_{j}$. The matrices $A, B$ under the similarity by $U$ take the form

$$
\left[\begin{array}{ll}
\lambda_{i} & \\
& \tilde{\lambda}
\end{array}\right] \oplus A_{0} \text { and }\left[\begin{array}{ll}
\tilde{\mu} & \\
& \mu_{j}
\end{array}\right] \oplus B_{0},
$$

respectively.

Now let $\mathcal{L}$ be a 3 -dimensional subspace spanned by $x_{i}, y_{j}$ and yet another vector $x$ orthogonal to the first two. Then the compressions of $A$ and $B$ onto $\mathcal{L}$ with respect to the basis $\left\{x_{i}, y_{j}, x\right\}$ are diagonal matrices with the diagonal entries $\lambda_{i}, \tilde{\lambda}$, $x^{*} A_{0} x$ and $\tilde{\mu}, \mu_{j}, x^{*} B_{0} x$, respectively. Since the total power dominance is inherited by definition, we are now in the setting of Lemma 6 . According to this lemma,

$$
x^{*} B_{0} x=\alpha x^{*} A_{0} x+\beta,
$$

with

$$
\alpha=\frac{\mu_{j}-\tilde{\mu}}{\tilde{\lambda}-\lambda_{i}}, \quad \beta=\frac{\tilde{\mu} \tilde{\lambda}-\mu_{j} \lambda_{i}}{\tilde{\lambda}-\lambda_{i}} .
$$

Letting $x$ run through the unit sphere of $\mathbb{C}^{n-2}$, we conclude from (10) that $B_{0}=$ $\alpha A_{0}+\beta I$, and therefore $B=\alpha A+\beta I$. 
The transition to infinite dimensions is easy.

Proof of necessity; infinite-dimensional setting. It suffices to consider two selfadjoint operators $A, B$ such that $A$ totally spectral dominates $B$ while

$$
a=\min \sigma(A)<b=\max \sigma(B) .
$$

Pick a unit vector $x$ in the range of the spectral projection of $A$ corresponding to the interval $[a, a+\epsilon]$ and a unit vector $y$ in the range of the spectral projection of $B$ corresponding to the interval $[b-\epsilon, b]$ for some $\epsilon<(a+b) / 2$. These vectors are orthogonal, since $A \succcurlyeq \stackrel{S}{\xi} B$. On the other hand, $\langle A x, x\rangle<\langle B y, y\rangle$. Choosing any unit vector $z$ orthogonal to $x, y$ and letting $\mathcal{L}=\operatorname{span}\{x, y, z\}$, we conclude from the already-proved finite-dimensional version that

$$
T_{\mathcal{L}}(B)=\alpha T_{\mathcal{L}}(A)+\beta I .
$$

The values of $\alpha$ and $\beta$ are determined uniquely by the compressions of $A$ and $B$ onto $\operatorname{span}\{x, y\}$ and thus do not depend on the choice of $z$.

It follows that the numerical range of the compression of $B-\alpha A-\beta I$ onto $(\operatorname{span}\{x, y\})^{\perp}$ is zero. Consequently, the compression itself is equal to zero. Since, moreover, $(B-\alpha A-\beta I) z$ is orthogonal to $x, y$ for any $z$ as above, in fact the restriction of $B-\alpha A-\beta I$ onto $(\operatorname{span}\{x, y\})^{\perp}$ is zero. Combining this conclusion with (11) completes the proof.

Note that only compressions onto 2-dimensional subspaces were used in the proofs in the case of unitarily irreducible pairs of matrices, and 2- or 3-dimensional subspaces in the general case. Consequently, the following observation holds.

Corollary 8. Let $A, B$ be a pair of selfadjoint operators. Then $A$ totally spectral dominates $B$ if and only if $T_{\mathcal{L}}(A) \succcurlyeq T_{\mathcal{L}}(B)$ for all subspaces $\mathcal{L}$ of dimension not exceeding 3 (2, if $A, B$ is the pair of unitarily irreducible matrices).

The authors want to thank the referee who brought reference [1] to their attention.

\section{REFERENCES}

[1] R. Bhatia, Matrix analysis, Graduate Texts in Mathematics, vol. 169, Springer-Verlag, New York, 1997. MR1477662 (98i:15003)

[2] C. Davis, Separation of two linear subspaces, Acta Sci. Math. (Szeged) 19 (1958), 172-187. MR.0098980(20:5425)

[3] W. F. Donoghue, Jr., Monotone matrix functions and analytic continuation, SpringerVerlag, New York, 1974, Die Grundlehren der mathematischen Wissenschaften, Band 207. MR0486556 (58:6279)

[4] M. Fujii and I. Kasahara, A remark on the spectral order of operators, Proc. Japan Acad. 47 (1971), suppl. II, 986-988. MR0312311 (47:873)

[5] T. Furuta, $A \geq B \geq 0$ assures $\left(B^{r} A^{p} B^{r}\right)^{1 / q} \geq B^{(p+2 r) / q}$ for $r \geq 0, p \geq 0, q \geq 1$ with $(1+2 r) q \geq p+2 r$, Proc. Amer. Math. Soc. 101 (1987), no. 1, 85-88. MR897075 (89b:47028)

[6] Spectral order $A \succ B$ if and only if $A^{2 p-r} \geq\left(A^{-r / 2} B^{p} A^{-r / 2}\right)^{(2 p-r) /(p-r)}$ for all $p>r \geq 0$ and its application, Math. Inequal. Appl. 4 (2001), no. 4, 619-624. MR.1859666 (2002g:47029)

[7] R. Giles and H. Kummer, A matrix representation of a pair of projections in Hilbert space, Canad. Math. Bull. 14 (1971), no. 1, 35-44. MR0291822(45:912)

[8] K. E. Gustafson and D. K. M. Rao, Numerical range. The field of values of linear operators and matrices, Springer, New York, 1997. MR 1417493 (98b:47008)

[9] P. R. Halmos, Two subspaces, Trans. Amer. Math. Soc. 144 (1969), 381-389. MR0251519 $(40: 4746)$ 
[10] R. A. Horn and C. R. Johnson, Topics in matrix analysis, Cambridge University Press, Cambridge, 1991. MR1091716 (92e:15003)

[11] M. P. Olson, The selfadjoint operators of a von Neumann algebra form a conditionally complete lattice, Proc. Amer. Math. Soc. 28 (1971), 537-544. MR0276788 (43:2528)

[12] V. E. S. Szabó, A class of matrix monotone functions, Linear Algebra Appl. 420 (2007), 79-85. MR 2277630

Department of Mathematics, College of William \& Mary, Williamsburg, Virginia 23185

Current address: Department of Mathematics, University of Michigan, Ann Arbor, Michigan 48109-1043

E-mail address: bhoai@umich.edu

Department of Mathematics, College of William \& Mary, Williamsburg, Virginia 23185

E-mail address: crjohnso@math.wm.edu

Department of Mathematics, College of William \& Mary, Williamsburg, Virginia 23185

E-mail address: ilya@math.wm.edu 